\title{
Application of a multiplex urinalysis test for the prediction of intravesical BCG treatment response: A pilot study
}

\author{
Kaoru Murakami ${ }^{\mathrm{a}}$, Ashish M. Kamat ${ }^{\mathrm{b}}$, Yunfeng Dai ${ }^{\mathrm{c}}$, Ian Pagano ${ }^{\mathrm{d}}$, Runpu Chen ${ }^{\mathrm{e}}$, Yijun Sun ${ }^{\mathrm{e}, \mathrm{f}, \mathrm{g}}$, \\ Amit Gupta $^{\mathrm{h}}$, Steve Goodison ${ }^{\mathrm{i}}$, Charles J. Rosser ${ }^{\mathrm{a}, \mathrm{h}, \mathrm{j}}$ and Hideki Furuya ${ }^{\mathrm{a}, *}$ \\ ${ }^{a}$ Samuel Oschin Comprehensive Cancer Institute, Cedars-Sinai Medical Center, Los Angeles, CA, USA \\ ${ }^{\mathrm{b}}$ Department of Urology, UT MD Anderson Cancer Center, Houston, TX, USA \\ ${ }^{\mathrm{c}}$ Department of Epidemiology, University of Florida, Gainesville, FL, USA \\ ${ }^{\mathrm{d}}$ Cancer Prevention and Control Program, University of Hawaii Cancer Center, Honolulu, HI, USA \\ ${ }^{\mathrm{e}}$ Department of Microbiology and Immunology, The State University of New York at Buffalo, Buffalo, NY, USA \\ ${ }^{\mathrm{f}}$ Department of Computer Science and Engineering, The State University of New York at Buffalo, Buffalo, NY, USA \\ ${ }^{\mathrm{g}}$ Department of Biostatistics, The State University of New York at Buffalo, Buffalo, NY, USA \\ ${ }^{\mathrm{h}}$ Division of Urology, Cedars-Sinai Medical Center, Los Angeles, CA, USA \\ ${ }^{i}$ Quantitative Health Sciences, Mayo Clinic, Jacksonville, FL, USA \\ ${ }^{\mathrm{i}}$ Nonagen Bioscience Corp., Los Angeles, CA, USA
}

Received 16 April 2021

Accepted 6 August 2021

\begin{abstract}
.
BACKGROUND: Intravesical Bacillus Calmette-Guerin (BCG), a live attenuated tuberculosis vaccine that acts as a non-specific immune system stimulant, is the most effective adjuvant treatment for patients with intermediate or high-risk non-muscle-invasive bladder cancer (NMIBC). However, to date, there are no reliable tests that are predictive of BCG treatment response. In this study, we evaluated the performance of Oncuria ${ }^{\mathrm{TM}}$, a bladder cancer detection test, to predict response to intravesical BCG.

METHODS: Oncuria ${ }^{\mathrm{TM}}$ data was evaluated in voided urine samples obtained from a prospectively collected cohort of 64 subjects with intermediate or high risk NMIBC prior to treatment with intravesical BCG. The Oncuria ${ }^{\mathrm{TM}}$ test, which measures 10 cancerassociated biomarkers was performed in an independent clinical laboratory. The ability of the test to identify those patients in whom BCG is ineffective against tumor recurrence was tested. Predictive models were derived using supervised learning and cross-validation analyses. Model performance was assessed using ROC curves.

RESULTS: Pre-treatment urinary concentrations of MMP9, VEGFA, CA9, SDC1, PAI1, APOE, A1AT, ANG and MMP10 were increased in patients who developed disease recurrence. A combinatorial predictive model of treatment outcome achieved an AUROC 0.89 [95\% CI: 0.80-0.99], outperforming any single biomarker, with a test sensitivity of $81.8 \%$ and a specificity of $84.9 \%$. Hazard ratio analysis revealed that patients with higher urinary levels of ANG, CA9 and MMP10 had a significantly higher risk of disease recurrence.

CONCLUSIONS: Monitoring the urinary levels of a cancer-associated biomarker panel enabled the discrimination of patients who did not respond to intravesical BCG therapy. With further study, the multiplex Oncuria ${ }^{\mathrm{TM}}$ test may be applicable for the clinical evaluation of bladder cancer patients considering intravesical BCG treatment.
\end{abstract}

Keywords: Biomarkers, bladder cancer, multiplex, protein, BCG

\footnotetext{
*Corresponding author: Hideki Furuya, Samuel Oschin Comprehensive Cancer Institute, Cedars-Sinai Medical Center, 110 N. George
}

Burns Road, Davis 2025, Los Angeles, CA, 90048, USA. Tel.: +1 310423 4607; E-mail: hideki.furuya@cshs.org. 


\section{Introduction}

Up to $60 \%$ of non-muscle invasive bladder cancer (NMIBC) cases that are treated by transurethral resection (TUR) will experience disease recurrence. Guidelines for NMIBC management include the recommendation for post-TUR intravesical instillation therapy [1,2]. Intravesical Bacillus Calmette-Guerin (BCG), a live attenuated tuberculosis vaccine that acts as a nonspecific immune system stimulant, has proven to be the most successful adjuvant treatment to date, assisting in the eradication of residual disease, reducing recurrence rates, and decreasing disease progression to muscleinvasive bladder cancer (MIBC) [1,2]. However, despite considerable success, as many as $30 \%$ of patients will develop tumor recurrence and up to $15 \%$ can progress despite BCG therapy $[3,4]$. Failure to intervene with definitive radical cystectomy prior to progression to MIBC is associated with a significant reduction in longterm survival probability $[5,6]$. Thus, early identification of patients suited for bladder preservation with BCG treatment or for radical cystectomy is essential. At this time, the decision to preserve the bladder or to perform a cystectomy depends on models based on clinicopathological parameters $[7,8]$, but these tools have limited accuracy for predicting disease recurrence or progression $[9,10]$. Furthermore, there is currently no established evaluation test available for the prediction of patient response to intravesical BCG.

Previously, we have identified a panel of protein biomarkers that are associated with bladder cancer [1114], and we have developed a multiplex immunoassay for the automated detection of the analyte panel in voided urine [15-17]. The test has been validated for non-invasive diagnosis, but in this study, we tested the potential clinical utility of the multiplex test for the prediction of BCG treatment response in a small prospective cohort.

\section{Patients and methods}

\subsection{Patients, specimens and data collection}

Patients with intermediate and high risk NMIBC (Tis, Ta or T1) [18] were previously recruited and reported in a clinical trial in which intravesical BCG was administered [19]. Briefly, spontaneous voided urine samples were collected prior to BCG treatment. All urine samples were centrifuged at $1,500 \mathrm{~g}$ for $10 \mathrm{~min}$, and cell-free urine samples were stored at $-80^{\circ} \mathrm{C}$ prior to analysis. After the initiation of intravesical BCG treatment, all participants were followed up with periodic cystoscopic medical examination. The endpoint of interest in each patient was disease recurrence, defined as a newly identified bladder tumor after a previous negative follow-up cystoscopy. Patients with noted abnormality on cystoscopy, underwent cystoscopy with bladder biopsy or transurethral resection of a bladder tumor (TURBT) followed by pathological interpretation.

\subsection{Multiplex immunoassay}

The concentrations of the 10 proteins (A1AT, APOE, ANG, CA9, IL8, MMP9, MMP10, PAI1, SDC1 and VEGFA) were monitored using an analytically validated multiplex bead-based immunoassay (Oncuria ${ }^{\mathrm{TM}}$ ) from R\&D Systems Inc. (Minneapolis, MN) [15-17]. Urine samples were passively thawed on ice, centrifuged for 10 minutes $\times 1,000 \mathrm{~g}$ and handled on ice prior to diluting 2-fold with R\&D Assay Diluent. Samples, standards and controls $(50 \mu \mathrm{l})$ were added to the 96 well plate in duplicate. The multiplex immunoassay was conducted according to the manufacturer's instructions. A seven-point standard curve across the dynamic range of the assays was included in the current assay design. Plates were read on the Luminex ${ }^{\circledR} 200$ plate reader (Luminex Corp, Austin, TX). Calibration curves were generated along with optimal fit in conjunction with Akaike's information criteria (AIC) values [20].

\subsection{Data analysis}

Wilcoxon rank sum tests were used to determine the association between each biomarker and bladder cancer recurrence. Nonparametric receiver operating characteristic (ROC) curves were generated to plot assay sensitivity against the false-positive rate (1-specificity). The relative ability of each biomarker to predict bladder cancer recurrence was evaluated by calculating the area under the curve (AUC), and AUCs were compared by chi-square test. The sensitivity and specificity of each biomarker individually and in combination were estimated at the optimal cutoff value defined by the Youden index [21]. To assess the independent association between biomarkers and bladder cancer recurrence, we used logistic regression analysis with recurrence status (yes vs. no) as the response variable and biomarker concentrations as explanatory variables. Multivariate analysis using Cox proportional hazards models for recurrence was performed to evaluate the influences of each biomarker on disease-specific survival. The 
all-subset method was used to evaluate the predictive value of each possible combination of biomarkers, and the Bayesian information criterion (BIC) was used to compare models. The BIC, a widely used criterion in model selection, balances the model likelihood and the number of biomarkers included in the model [22]. The Bootstrap method (using 1000 Bootstrap samples) was used [23] to select the most efficient and stable predictive model. Statistical significance in this study was set at $p<0.05$ and all reported $p$ values were 2 -sided. All analyses were performed using SAS software version 9.3 (SAS Institute Inc., Cary, NC).

\section{Results}

The study population was comprised of 64 subjects with NMIBC who were scheduled to be treated with intravesical BCG. Among the 64 study subjects in this application, all 64 had high-grade disease with 23 with intermediate risk disease (i.e., ALL Ta high-grade < $3 \mathrm{~cm}$ ) and the remaining 41 high risk disease (i.e., 29 with high-grade T1, 7 with high-grade $\mathrm{Ta}>3 \mathrm{~cm}$ and 5 with CIS). The mean age of subjects was $65.8 \pm 11.3$ years, $78.1 \%$ of the subjects were men, $85.9 \%$ were Caucasian, and $54.7 \%$ of the subjects presented with Tis/Ta disease while $45.3 \%$ of the subjects presented with T1 disease (Table 1). Of the total 64 subjects, 11 $(15.6 \%)$ were found to have post-treatment bladder cancer recurrence on follow-up. Median time to recurrence was 6 months (range 1-17 months). The recurrences were noted to be non-muscle invasive bladder cancer (NMIBC; stages Ta, Tis, T1) high-grade in $81.8 \%$ ( $n=$ 9 ), and muscle invasive bladder cancer (MIBC; stage $\geqslant$ T2) high-grade in $18.2 \%(n=2)$. Of these 11 subjects, two were noted to have a second recurrence.

For each of the 64 clinical samples, we reported the mean \pm SD and range of each biomarker, along with the percentage of samples in which the biomarker was detectable (Table 2). Each individual biomarker was detectable in $>90 \%$ of the samples, except for A1AT (detected in $58 \%$ of samples). Increased urinary concentrations of MMP9 $(P=0.53)$, VEFGA $(P=0.20)$, CA9 $(P=0.19), \operatorname{SDC} 1(P=0.23)$, PAI1 $(P=0.19)$, $\operatorname{ApoE}(P=0.21), \operatorname{A1AT}(P=0.04), \operatorname{ANG}(P=0.02)$ and MMP10 $(P=0.15)$ were observed in subjects with disease recurrence. The urinary concentration of IL8 was unchanged.

Table 3 provides AUC data for each individual biomarker and for the combination of all 10 biomarkers in the Oncuria ${ }^{\mathrm{TM}}$ test. Using optimal cutoff values
Table 1

Demographic and clinical-pathologic characteristics of study cohorts

\begin{tabular}{|c|c|c|c|c|c|c|}
\hline \multirow[b]{2}{*}{ Variable } & \multirow[b]{2}{*}{ Value } & \multicolumn{2}{|c|}{$\begin{array}{l}\text { No recurrence } \\
\quad(N=53)\end{array}$} & \multicolumn{2}{|c|}{$\begin{array}{l}\text { Recurrence } \\
(N=11)\end{array}$} & \multirow[b]{2}{*}{$P$} \\
\hline & & $n$ & $\%$ & $n$ & $\%$ & \\
\hline \multirow[t]{4}{*}{ Age } & $18-54$ & 9 & 17.0 & 2 & 18.2 & 0.37 \\
\hline & $55-64$ & 16 & 30.2 & 4 & 36.4 & \\
\hline & $65-74$ & 17 & 32.1 & 1 & 9.1 & \\
\hline & $75+$ & 11 & 20.8 & 4 & 36.4 & \\
\hline \multirow[t]{2}{*}{ Sex } & Female & 10 & 18.9 & 4 & 36.4 & 0.22 \\
\hline & Male & 43 & 81.1 & 7 & 63.6 & \\
\hline \multirow[t]{2}{*}{ Race } & White & 46 & 86.8 & 9 & 81.8 & 0.67 \\
\hline & Other & 7 & 13.2 & 2 & 18.2 & \\
\hline \multirow[t]{3}{*}{ Stage } & $\mathrm{Ta}$ & 26 & 49.1 & 4 & 36.4 & 0.21 \\
\hline & Tis & 5 & 9.4 & 0 & 0.0 & \\
\hline & $\mathrm{T} 1$ & 22 & 41.5 & 7 & 63.6 & \\
\hline \multirow[t]{3}{*}{ Cytology } & Negative & 24 & 45.3 & 2 & 18.2 & 0.10 \\
\hline & Positive & 25 & 47.2 & 6 & 54.5 & \\
\hline & Missing & 4 & 7.5 & 3 & 27.3 & \\
\hline
\end{tabular}

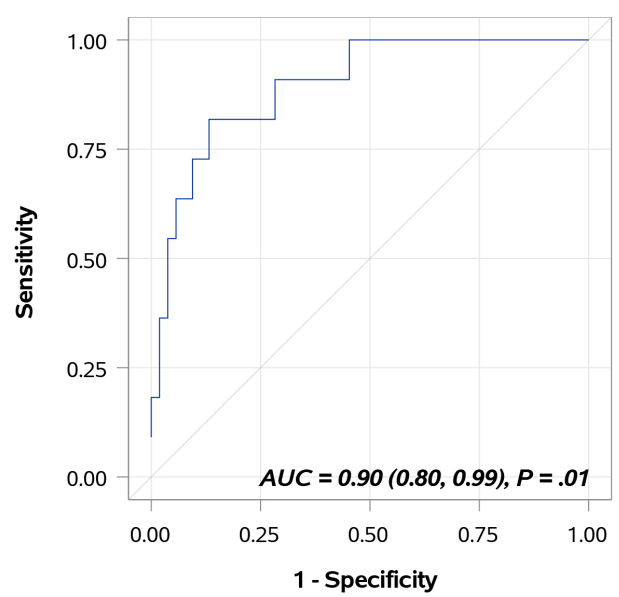

Fig. 1. Diagnostic performance of bladder cancer-associated molecular signature. The areas under the curves was 0.8971 (95\% confidence interval, $0.8000-0.9942$ ), with a sensitivity value of $81.8 \%$ and a specificity value of $84.9 \%$ for the prediction of disease recurrence.

defined by the Youden index from this cohort, the 10biomarker model resulted in an AUC of 0.8971 (95\% confidence interval, 0.8000-0.9942), with a sensitivity value of $81.8 \%$ and a specificity value of $84.9 \%$ for the prediction of disease recurrence (Fig. 1). Patients with higher urinary levels of CA9 (HR: $3.44,95 \% \mathrm{CI}$ : $1.21-9.76 ; P=0.02)$, ANG (HR: 42.89 , 95\% CI: 3.06 602.10; $P=0.005$ ) and MMP10 (HR: $3.86,95 \% \mathrm{CI}$ : $1.06-14.05 ; P=0.04$ ) had a significantly higher risk of disease recurrence. Increased levels of PAI1 (HR: 3.54, 95\% CI: 0.87-14.43; $P=0.08$ ), APOE (HR: $4.64,95 \%$ CI: $0.91-23.59 ; P=0.06$ ) and A1AT (HR: 3.72, 95\% CI: $0.94-14.83 ; P=0.06)$ approached significance (Table 4). 
Table 2

Mean urinary $\left( \pm\right.$ SD) concentrations of 10 biomarkers assessed by Oncuria ${ }^{\mathrm{TM}}$

\begin{tabular}{|c|c|c|c|c|c|c|c|c|c|c|c|}
\hline \multirow{2}{*}{$\begin{array}{l}\text { Biomarker } \\
\mathrm{pg} / \mathrm{mL}\end{array}$} & \multirow[t]{2}{*}{$\begin{array}{c}\text { Detectable } \\
\%\end{array}$} & \multicolumn{4}{|c|}{ No recurrence $(N=53)$} & \multicolumn{4}{|c|}{ Recurrence $(N=11)$} & \multirow[b]{2}{*}{$P$} & \multirow[b]{2}{*}{ Cutoff } \\
\hline & & Mean & SD & Min & Max & Mean & SD & Min & Max & & \\
\hline MMP-9 & 92 & 10,873 & 12,604 & 292 & 60,355 & 13,986 & 14,885 & 653 & 39,466 & 0.53 & $>6,051$ \\
\hline CXCL8/IL-8 & 100 & 425.8 & 373.6 & 6.4 & $1,508.1$ & 417.2 & 395.4 & 39.4 & $1,322.3$ & 0.95 & $<254.4$ \\
\hline VEGF-A & 100 & 209.9 & 114.6 & 40.6 & 496.7 & 327.6 & 279.7 & 86.0 & 900.5 & 0.20 & $>209.3$ \\
\hline IX/CA9 & 91 & 2.07 & 3.46 & 0.07 & 20.81 & 6.87 & 11.31 & 0.32 & 32.32 & 0.19 & $>1.55$ \\
\hline Syndecan-1 & 100 & 6,580 & 2,860 & 1,256 & 14,433 & 7,911 & 3,294 & 3,341 & 12,176 & 0.23 & $>6,562$ \\
\hline Serpin E1/PAI-1 & 100 & 448.7 & 439.5 & 35.7 & $1,990.0$ & 955.7 & $1,196.0$ & 78.4 & $3,827.0$ & 0.19 & $>378.0$ \\
\hline ApoE & 100 & 8,714 & 9,513 & 676 & 53,748 & 12,925 & 9,763 & 2,555 & 34,151 & 0.21 & $>7,664$ \\
\hline Serpin A1 & 58 & 771,434 & 716,725 & 38,750 & $1,940,791$ & $1,257,290$ & 645,741 & 66,035 & $1,631,774$ & 0.04 & $>629,949$ \\
\hline Angiogenin & 100 & 2,284 & 1,323 & 24 & 6,718 & 4,179 & 2,339 & 1,185 & 8,603 & 0.02 & $>2,683$ \\
\hline MMP-10 & 95 & 236.6 & 286.5 & 7.5 & $1,341.3$ & 405.9 & 346.5 & 84.7 & $1,059.6$ & 0.15 & $>192.1$ \\
\hline
\end{tabular}

Table 3

Performance of the Oncuria ${ }^{\mathrm{TM}}$ test for the prediction of BCG treatment response

\begin{tabular}{|c|c|c|c|c|c|c|c|c|c|c|}
\hline Biomarker & $\begin{array}{l}\text { Area under } \\
\text { the curve }\end{array}$ & $\begin{array}{c}95 \% \text { confidence } \\
\text { interval }\end{array}$ & $\begin{array}{l}\text { No. of } \\
\text { correctly } \\
\text { predicted } \\
\text { events }\end{array}$ & $\begin{array}{c}\text { No. of } \\
\text { correctly } \\
\text { predicted } \\
\text { nonevents }\end{array}$ & $\begin{array}{c}\text { No. of } \\
\text { nonevents } \\
\text { predicted as } \\
\text { events }\end{array}$ & $\begin{array}{c}\text { No. of } \\
\text { events } \\
\text { predicted as } \\
\text { nonevents }\end{array}$ & Sensitivity & Specificity & PPV & NPV \\
\hline A1AT & 0.6364 & $(0.4606,0.8121)$ & 9 & 30 & 23 & 2 & 0.818 & 0.566 & 0.281 & 0.938 \\
\hline ANG & 0.7444 & $(0.5700,0.9189)$ & 7 & 43 & 10 & 4 & 0.636 & 0.811 & 0.412 & 0.915 \\
\hline APOE & 0.6724 & $(0.4985,0.8463)$ & 9 & 27 & 26 & 2 & 0.818 & 0.509 & 0.257 & 0.931 \\
\hline CA9 & 0.6878 & $(0.5203,0.8553)$ & 7 & 37 & 16 & 4 & 0.636 & 0.698 & 0.304 & 0.902 \\
\hline IL8 & 0.5146 & $(0.3146,0.7146)$ & 4 & 41 & 12 & 7 & 0.364 & 0.774 & 0.250 & 0.854 \\
\hline MMP9 & 0.542 & $(0.3469,0.7371)$ & 10 & 14 & 39 & 1 & 0.909 & 0.264 & 0.204 & 0.933 \\
\hline MMP10 & 0.7238 & $(0.5787,0.8690)$ & 8 & 36 & 17 & 3 & 0.727 & 0.679 & 0.320 & 0.923 \\
\hline PAI1 & 0.6261 & $(0.4306,0.8215)$ & 7 & 35 & 18 & 4 & 0.636 & 0.660 & 0.280 & 0.897 \\
\hline SDC1 & 0.6106 & $(0.3927,0.8286)$ & 6 & 47 & 6 & 5 & 0.545 & 0.887 & 0.500 & 0.904 \\
\hline VEGFA & 0.5935 & $(0.3904,0.7965)$ & 3 & 52 & 1 & 8 & 0.273 & 0.981 & 0.750 & 0.867 \\
\hline $\begin{array}{l}\text { 10-biomarker } \\
\text { combination }\end{array}$ & 0.8971 & $(0.8000,0.9942)$ & 9 & 45 & 8 & 2 & 0.818 & 0.849 & 0.529 & 0.957 \\
\hline
\end{tabular}

Table 4

Hazard ratio of tumor recurrence based on molecular signature

\begin{tabular}{lrrrl}
\hline $\begin{array}{c}\text { Biomarker } \\
(\log 10 \mathrm{pg} / \mathrm{ml})\end{array}$ & HR & LCL & UCL & $P$-value \\
\hline MMP-9 & 1.36 & 0.48 & 3.84 & 0.56 \\
CXCL8/IL-8 & 0.97 & 0.31 & 3.03 & 0.96 \\
VEGF-A & 7.38 & 0.68 & 80.43 & 0.10 \\
IX/CA9 & 3.44 & 1.21 & 9.76 & $\mathbf{0 . 0 2}$ \\
Syndecan-1 & 6.82 & 0.29 & 159.23 & 0.23 \\
Serpin E1/PAI-1 & 3.54 & 0.87 & 14.43 & 0.08 \\
ApoE & 4.64 & 0.91 & 23.59 & 0.06 \\
Serpin A1 & 3.72 & 0.94 & 14.83 & 0.06 \\
Angiogenin & 42.89 & 3.06 & 602.10 & $\mathbf{0 . 0 0 5}$ \\
MMP-10 & 3.86 & 1.06 & 14.05 & $\mathbf{0 . 0 4}$ \\
\hline
\end{tabular}

\section{Discussion}

A comprehensive review of the literature regarding potential predictive biomarkers of BCG response reveals limited results. In 2003, investigators reported that the failure to detect urinary IL-2 during a BCG induction course correlated with the time to disease recurrence and progression [24]. Similarly, Kamat and others developed the CyPRIT assay and noted that an increase in nine cytokines, including IL-2, induced after a BCG treatment correlated with disease recurrence, and that combinatorial changes in the cytokine panel could best predict disease recurrence [19]. Monitoring cytokine profile changes is promising as a test of immune response once BCG treatment is initiated and may guide BCG treatment frequency or continuance in the individual.

Previous studies have assessed the ability of the diagnostic FISH test (UroVysionTM, Abbott Molecular Inc.) to predict whether patients with NMIBC would incur disease recurrence or progression after BCG treatment. UroVysion testing was applied at the beginning and the end of the BCG induction cycle (typically involving 6 weekly instillations), and urine cytology and cystoscopy were performed six weeks after cycle completion. In a multivariate analysis, the presence of highgrade disease and a positive UroVysion test after BCG initiation were significant predictors of disease recurrence [25,26]. Again, in these studies, the urine-based results were only informative after the initiation of BCG treatment, and so are not predictive of BCG outcome 
Table 5

Annotated urine-based bladder cancer associated diagnostic

\begin{tabular}{|c|c|c|c|c|}
\hline Full name & Abbreviation & Ascribed function & Location & $\begin{array}{c}\text { Interacts with } \\
\text { other members } \\
\text { of signature }\end{array}$ \\
\hline Interleukin 8 & IL8 & Chemoattractant \& angiogenesis & Extracellular & MMP9, SDC1 \\
\hline Angiogenin & ANG & Angiogenesis & Extracellular, nucleus & None \\
\hline $\begin{array}{l}\text { Vascular endothelial growth } \\
\text { factor A }\end{array}$ & VEGFA & Angiogenesis & Extracellular, cytoplasm & None \\
\hline Matrix metallopeptidase 9 & MMP9 & Breakdown of extracellular matrix & Extracellular & IL8, MMP10 \\
\hline Matrix metallopeptidase 10 & MMP10 & Breakdown of extracellular matrix & Extracellular & MMP9 \\
\hline Serpin peptidase inhibitor & SERPINA1 & Serine protease inhibitor & Extracellular & None \\
\hline Serpin peptidase inhibitor & SERPINE1 & Serine endopeptidase inhibitor & Extracellular, plasma membrane & None \\
\hline Carbonic anhydrase IX & CA9 & $\begin{array}{l}\text { Catalyze the reversible hydration of carbon } \\
\text { dioxide }\end{array}$ & Plasma membrane & None \\
\hline Apolipoprotein E & APOE & Lipoprotein catabolism and metabolism & $\begin{array}{l}\text { Extracellular, plasma membrane, } \\
\text { cytoplasm }\end{array}$ & None \\
\hline Syndecan 1 & SDC1 & $\begin{array}{l}\text { Cell binding, cell signaling, cytoskeletal } \\
\text { organization }\end{array}$ & Plasma membrane, cytoplasm & IL8 \\
\hline
\end{tabular}

prior to the first cycle of instillation. Therefore, the information would not be available to guide the clinical management of the individual patient regarding the decision to embark on an intravesical BCG treatment regimen. In a small prospective study, Lotan and others noted a positive FISH test prior to BCG to be associated with recurrence and progression, hazard ratio HR 2.59 (95\% CI 1.42-4.73) [27].

Here, we evaluated the performance of the diagnostic Oncuria $^{\mathrm{TM}}$ test to predict the response to BCG therapy, prior to treatment initiation. In this pilot study, we modified the established diagnostic algorithm, which weights biomarker values to produce a risk score, to the fit the predictive scenario. The 10 biomarkers that comprise the Oncuria ${ }^{\mathrm{TM}}$ test were reliably detected in almost all of the 64 urine samples, and the levels of 9 of the biomarkers were elevated in pre-treatment urine samples from subjects who had a subsequent bladder cancer recurrence after the completion of BCG treatment. As shown in diagnostic applications, it is the combination of the biomarker values through a weighted analytical algorithm that provides a model with sufficient predictive power for potential clinical application. In this case, the test shows promise for the evaluation of patients with respect to the decision to undergo BCG treatment.

The biomarkers that compose the bladder cancer diagnostic signature have a varied range of reported functions including angiogenesis, breakdown of extracellular matrix, serine protein inhibitor, catalyze the reversible hydration of carbon dioxide, lipoprotein metabolism and cell binding/signaling (Table 5) with the two primary functional groups being extracellular matrix remodeling (MMP9 and MMP10) and angiogenesis (IL8, VEGFA and ANG). Angiogenesis, the development of new blood vessels from existing blood vessels, is essential for normal growth and development of tissues and organs. A balance of pro-angiogenic factors and anti-angiogenic factors tightly controls this process [28-30]. However in solid tumors, the balance may favor pro-angiogenic factors, ensuring nutrients are provided to the rapidly dividing cancer cells, thus allowing the support of the abnormal growth seen in tumors [31]. Recent studies also suggest ANG and PAI1 can breakdown the extracellular matrix [32,33], allowing cancer cells to invade and metastasize [34]. The extent of tumor vascularization differs between malignancies, and has been shown to correlate directly with metastatic potential [35].

We recognize that the study has several limitations. First, as a pilot study, with only 64 total subjects the study is small and underpowered. Second, defining truly independent disease recurrence and distinguishing new lesions from regrowth from a previous tumor resection site can be challenging. In future studies, we would propose noting the location of the primary tumor, as well as the location and timing of the recurrence, realizing that a second tumor within the previous tumor bed at 3 months follow-up may not necessarily be due to a lack of BCG response, while recurrences outside of the tumor bed may be more indicative of BCG failure. The evaluation of the test in a larger, prospective cohort with more information on lesion characteristics before and after BCG treatment may determine whether the urinary biomarker profile indicates residual disease or a field change in the urothelial or immune landscape which may be predictive of the response to intravesical BCG.

The development of an accurate and robust test that can predict BCG treatment response would benefit both 
patients and healthcare systems. The identification of predictive molecular signatures has the potential to better tailor treatment regimens for individual patients, avoiding potentially significant delays in clinical management. In this pilot study, the multiplex Oncuria ${ }^{\mathrm{TM}}$ test achieved encouraging predictive performance. The test uses established technology facilitating uptake in clinical laboratories once validated for defined applications. Additional studies are underway to evaluate the potential added value of the test in clinical decision making.

\section{Ethics approval and consent to participate}

MD Anderson Cancer Center local ethics review board approved.

\section{Consent for publication}

Not applicable.

\section{Availability of data and materials}

Reasonable requests for data will be made available for review.

\section{Competing interests}

Dr. Charles Rosser is an officer of Nonagen Bioscience. No financial or commercial conflicts of interest were declared by other co-authors.

\section{Conflict of interest}

CJR is an officer of Nonagen Bioscience Corporation.

\section{Funding}

This work was supported by research grants R01 CA1988887 (CJR) and R01 CA206584 (SG).

\section{Author contributions}

Conception, interpretation or analysis of data: KM. Interpretation or analysis of data, revision for important intellectual content: AK.

Interpretation or analysis of data: IP, RC, YS.

Revision for important intellectual content: AG and SG.
Conception, supervision, preparation of the manuscript: CJR and HF.

\section{References}

[1] M.C. Hall, S.S. Chang, G. Dalbagni, R.S. Pruthi, J.D. Seigne, E.C. Skinner, J.S. Wolf, Jr. and P.F. Schellhammer, Guideline for the management of nonmuscle invasive bladder cancer (stages Ta, T1, and Tis): 2007 update, J Urol 178 (2007), 2314-2330.

[2] M. Babjuk, M. Burger, R. Zigeuner, S.F. Shariat, B.W. van Rhijn, E. Comperat, R.J. Sylvester, E. Kaasinen, A. Bohle, J. Palou Redorta, M. Roupret and U. European Association of, EAU guidelines on non-muscle-invasive urothelial carcinoma of the bladder: Update 2013, Eur Urol 64 (2013), 639-653.

[3] J.A. Witjes, Management of BCG failures in superficial bladder cancer: A review, Eur Urol 49 (2006), 790-797.

[4] J.T. Matulay, R. Li, P.J. Hensley, N.A. Brooks, V.M. Narayan, H.B. Grossman, N. Navai, C.P.N. Dinney and A.M. Kamat Contemporary outcomes of patients with nonmuscle-invasive bladder cancer treated with bacillus Calmette-Guerin: Implications for clinical trial design, J Urol 205 (2021), 1612-1621.

[5] F.C. von Rundstedt and S.P. Lerner, Bacille-Calmette-Guerin non-responders: How to manage, Transl Androl Urol 4 (2015), 244-253.

[6] A. Gupta, Y. Lotan, P.J. Bastian, G.S. Palapattu, P.I. Karakiewicz, G.V. Raj, M.P. Schoenberg, S.P. Lerner, A.I Sagalowsky, S.F. Shariat and C. Bladder Cancer Research, Outcomes of patients with clinical T1 grade 3 urothelial cell bladder carcinoma treated with radical cystectomy, Urology 71 (2008), 302-307.

[7] T. Yamada, K. Tsuchiya, S. Kato, S. Kamei, M. Taniguchi, T Takeuchi, N. Yamamoto, H. Ehara and T. Deguchi, A pretreatment nomogram predicting recurrence- and progression-free survival for nonmuscle invasive bladder cancer in Japanese patients, Int J Clin Oncol 15 (2010), 271-279.

[8] A.M. Kamat, R. Li, M.A. O’Donnell, P.C. Black, M. Roupret, J.W. Catto, E. Comperat, M.A. Ingersoll, W.P. Witjes, D.J. McConkey and J.A. Witjes, Predicting response to intravesical bacillus Calmette-Guerin immunotherapy: Are we there yet? A systematic review, Eur Urol 73 (2018), 738-748.

[9] R.J. Lammers, J.C. Hendriks, O.R. Rodriguez Faba, W.P. Witjes, J. Palou and J.A. Witjes, Prediction model for recurrence probabilities after intravesical chemotherapy in patients with intermediate-risk non-muscle-invasive bladder cancer, including external validation, World J Urol 34 (2016), 173-180.

[10] T.C. Zuiverloon, A.J. Nieuweboer, H. Vekony, W.J. Kirkels, C.H. Bangma and E.C. Zwarthoff, Markers predicting response to bacillus Calmette-Guerin immunotherapy in high-risk bladder cancer patients: A systematic review, Eur Urol 61 (2012), 128-145.

[11] N. Yang, S. Feng, K. Shedden, X. Xie, Y. Liu, C.J. Rosser, D.M. Lubman and S. Goodison, Urinary glycoprotein biomarker discovery for bladder cancer detection using LC/MS-MS and label-free quantification, Clin Cancer Res 17 (2011), 3349-3359.

[12] P. Kreunin, J. Zhao, C. Rosser, V. Urquidi, D.M. Lubman and S. Goodison, Bladder cancer associated glycoprotein signatures revealed by urinary proteomic profiling, J Proteome Res 6 (2007), 2631-2639.

[13] C.J. Rosser, L. Liu, Y. Sun, P. Villicana, M. McCullers, S. Porvasnik, P.R. Young, A.S. Parker and S. Goodison, Bladder 
cancer-associated gene expression signatures identified by profiling of exfoliated urothelia, Cancer Epidemiol Biomarkers Prev 18 (2009), 444-453.

[14] V. Urquidi, S. Goodison, Y. Cai, Y. Sun and C.J. Rosser, A candidate molecular biomarker panel for the detection of bladder cancer, Cancer Epidemiol Biomarkers Prev 21 (2012), 2149-2158

[15] H. Furuya, I. Pagano, K. Chee, T. Kobayashi, R.S. Wong, R Lee and C.J. Rosser, Comparison of commercial ELISA kits, a prototype multiplex electrochemoluminescent assay, and a multiplex bead-based immunoassay for detecting a urine-based bladder-cancer-associated diagnostic signature, Diagnostics (Basel) 9 (2019).

[16] H. Furuya, L. Tabula, R. Lee, P. Kralovec, M. Ramsden, R. Wong and C.J. Rosser, Analytical validation of ONCURIA a multiplex bead-based immunoassay for the non-invasive bladder cancer detection, Pract Lab Med 22 (2020), e00189.

[17] Y. Hirasawa, I. Pagano, R. Chen, Y. Sun, Y. Dai, A. Gupta, S Tikhonenkov, S. Goodison, C.J. Rosser and H. Furuya, Diagnostic performance of Oncuria, a urinalysis test for bladder cancer, J Transl Med 19 (2021), 141

[18] S.S. Chang, S.A. Boorjian, R. Chou, P.E. Clark, S. Daneshmand, B.R. Konety, R. Pruthi, D.Z. Quale, C.R. Ritch, J.D. Seigne, E.C. Skinner, N.D. Smith and J.M. McKiernan, Diagnosis and treatment of non-muscle invasive bladder cancer: AUA/SUO guideline, J Urol 196 (2016), 1021-1029.

[19] A.M. Kamat, J. Briggman, D.L. Urbauer, R. Svatek, G.M. Nogueras Gonzalez, R. Anderson, H.B. Grossman, F. Prat and C.P. Dinney, Cytokine panel for response to intravesical therapy (CyPRIT): Nomogram of changes in urinary cytokine levels predicts patient response to bacillus Calmette-Guerin, Eur Urol 69 (2016), 197-200.

[20] H. Motulsky and A. Christopoulos, Fitting models to biological data using linear and nonlinear regression: a practical guide to curve fitting, Oxford University Press, 2004.

[21] R. Fluss, D. Faraggi and B. Reiser, Estimation of the Youden Index and its associated cutoff point, Biom J 47 (2005), 458472.

[22] L.F. Leon and C.L. Tsai, Assessment of model adequacy for Markov regression time series models, Biometrics 54 (1998), 1165-1175.

[23] P.C. Austin and J.V. Tu, Bootstrap methods for developing predictive models, The American Statistician 58 (2004), 131137.

[24] F. Saint, N. Kurth, P. Maille, D. Vordos, A. Hoznek, P. Soyeux, J.J. Patard, C.C. Abbou and D.K. Chopin, Urinary IL-2 assay for monitoring intravesical bacillus Calmette-Guerin response of superficial bladder cancer during induction course and maintenance therapy, Int J Cancer 107 (2003), 434-440.
[25] J.M. Whitson, A.B. Berry, P.R. Carroll and B.R. Konety, UroVysion testing can lead to early identification of intravesical therapy failure in patients with high risk non-muscle invasive bladder cancer, Int Braz J Urol 35 (2009), 664-670; discussion 671-2.

[26] S. Savic, I. Zlobec, G.N. Thalmann, D. Engeler, M. Schmauss, K. Lehmann, G. Mattarelli, T. Eichenberger, P. Dalquen, P. Spieler, R. Schoenegg, T.C. Gasser, T. Sulser, T. Forster, T. Zellweger, R. Casella and L. Bubendorf, The prognostic value of cytology and fluorescence in situ hybridization in the followup of nonmuscle-invasive bladder cancer after intravesical Bacillus Calmette-Guerin therapy, Int J Cancer 124 (2009), 2899-2904

[27] Y. Lotan, B.A. Inman, L.G. Davis, W. Kassouf, E. Messing, S. Daneshmand, D. Canter, H.T. Marble, A.M. Joseph, S. Jewell and S.A. Boorjian, Evaluation of the fluorescence in situ hybridization test to predict recurrence and/or progression of disease after bacillus Calmette-Guerin for primary high grade nonmuscle invasive bladder cancer: Results from a prospective multicenter trial, J Urol 202 (2019), 920-926.

[28] J. Folkman, Angiogenesis in cancer, vascular, rheumatoid and other disease, Nat Med 1 (1995), 27-31.

[29] J. Folkman and M. Klagsbrun, Angiogenic factors, Science 235 (1987), 442-447.

[30] D. Hanahan and J. Folkman, Patterns and emerging mechanisms of the angiogenic switch during tumorigenesis, Cell $\mathbf{8 6}$ (1996), 353-364.

[31] C.H. Blood and B.R. Zetter, Tumor interactions with the vasculature: Angiogenesis and tumor metastasis, Biochim Biophys Acta 1032 (1990), 89-118.

[32] E.G. Giacoia, M. Miyake, A. Lawton, S. Goodison and C.J. Rosser, PAI-1 leads to G1-phase cell-cycle progression through cyclin D3/cdk4/6 upregulation, Mol Cancer Res 12 (2014), 322-334.

[33] M. Miyake, S. Goodison, A. Lawton, E. Gomes-Giacoia and C.J. Rosser, Angiogenin promotes tumoral growth and angiogenesis by regulating matrix metallopeptidase- 2 expression via the ERK1/2 pathway, Oncogene 34 (2015), 890-901.

[34] L.A. Liotta, J. Kleinerman and G.M. Saidel, Quantitative relationships of intravascular tumor cells, tumor vessels, and pulmonary metastases following tumor implantation, Cancer Res 34 (1974), 997-1004.

[35] N. Weidner, J. Folkman, F. Pozza, P. Bevilacqua, E.N. Allred, D.H. Moore, S. Meli and G. Gasparini, Tumor angiogenesis: A new significant and independent prognostic indicator in early-stage breast carcinoma, J Natl Cancer Inst 84 (1992), 1875-1887. 